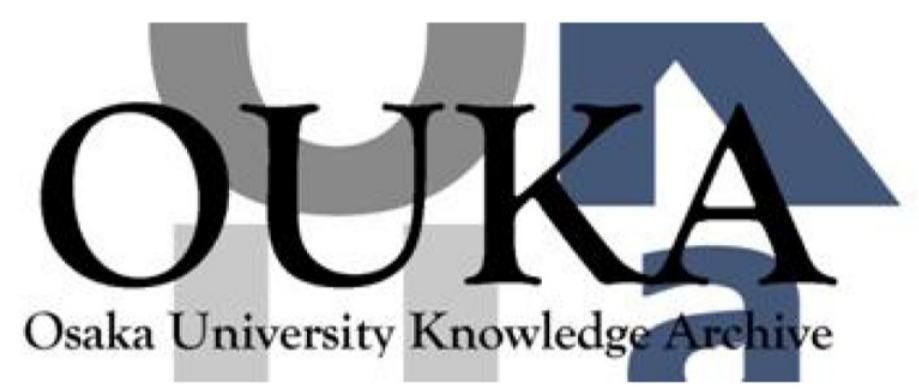

\begin{tabular}{|c|l|}
\hline Title & $\begin{array}{l}\text { Laser generated neutron source for neutron } \\
\text { resonance spectroscopy }\end{array}$ \\
\hline Author(s) & $\begin{array}{l}\text { Higginson, D.P.; McNaney, J.M. ; Swift, D. C. et } \\
\text { al. }\end{array}$ \\
\hline Citation & $\begin{array}{l}\text { Physics of Plasmas. 17(10) p. 100701-1-p. 100701- } \\
4\end{array}$ \\
\hline Issue Date & $2010-01$ \\
\hline oaire:version & VoR \\
\hline URL & https://hdl. handle. net/11094/3317 \\
\hline rights & \\
\hline Note & \\
\hline
\end{tabular}

Osaka University Knowledge Archive : OUKA

https://ir. Library. osaka-u. ac. jp/

Osaka University 


\title{
Laser generated neutron source for neutron resonance spectroscopy
}

\author{
D. P. Higginson, ${ }^{1,2}$ J. M. McNaney, ${ }^{2}$ D. C. Swift, ${ }^{2}$ T. Bartal, ${ }^{1,2}$ D. S. Hey, ${ }^{2}$ R. Kodama, ${ }^{3,4}$ \\ S. Le Pape, ${ }^{2}$ A. Mackinnon, ${ }^{2}$ D. Mariscal, ${ }^{1}$ H. Nakamura, ${ }^{3}$ N. Nakanii, ${ }^{1,3,4}$ \\ K. A. Tanaka, ${ }^{3,4}$ and F. N. Beg ${ }^{1}$ \\ ${ }^{1}$ Department of Mechanical and Aerospace Engineering, University of California-San Diego, La Jolla, \\ California 92093, USA \\ ${ }^{2}$ Lawrence Livermore National Laboratory, Livermore, California 94550, USA \\ ${ }^{3}$ Institute of Laser Engineering, Osaka University, 2-6, Yamada-oka, Suita, Osaka 565-0871, Japan \\ ${ }^{4}$ Graduate School of Engineering, Osaka University, 2-1, Yamada-oka, Suita, Osaka 565-0871, Japan
}

(Received 6 May 2010; accepted 9 August 2010; published online 14 October 2010)

\begin{abstract}
A neutron source for neutron resonance spectroscopy has been developed using high-intensity, short-pulse lasers. This technique will allow robust measurement of interior ion temperature of laser-shocked materials and provide insight into material equation of state. The neutron generation technique uses laser-accelerated protons to create neutrons in $\operatorname{LiF}$ through $(p, n)$ reactions. The incident proton beam has been diagnosed using radiochromic film. This distribution is used as the input for a $(p, n)$ neutron prediction code which is validated with experimentally measured neutron yields. The calculation infers a total fluence of $1.8 \times 10^{9}$ neutrons, which are expected to be sufficient for neutron resonance spectroscopy temperature measurements. (C) 2010 American Institute of Physics. [doi:10.1063/1.3484218]
\end{abstract}

A concrete understanding of material equation of state (EOS) is of underlying importance in many fields (e.g., planetary physics, geophysics, shocked matter physics, and warm-dense matter physics). One of the most fundamental quantities in EOS measurement is temperature, which is rarely measured. A major hurdle to temperature measurements is that conventional optical diagnostic techniques cannot be used to probe the interior of opaque materials, such as metals, while surface or interface techniques are inherently complex. A technique known as neutron resonance spectroscopy ${ }^{1}$ (NRS) avoids this problem by using neutrons to diagnose temperature.

In NRS, a beam of neutrons, with energies of $1-100 \mathrm{eV}$, is sent through a sample made of or doped with a material possessing strong neutron absorption resonances at these energies (e.g., W and Mo). When passing through, the material neutrons are absorbed by nuclear resonances in the ions, which can be seen as negative-peaks in the resulting neutron spectrum. From these peaks, the spectral broadening and shifting can be measured, enabling determination of ion temperature and velocity, respectively.

So far, NRS experiments have been performed using spallation neutrons from a proton accelerator ring. These experiments probed a shocked Mo target doped with W and inferred a corrected temperature ${ }^{2}$ of around $700 \mathrm{~K}$ with error bars of $\pm 100 \mathrm{~K}$, these error bars are lower than currently demonstrated with other nonoptical measurement techniques (e.g., x-ray Thomson scattering). However, in order to provide shocked states for EOS investigation at these facilities, detonation of chemical explosives accelerates flyer plates into the material of interest. This technique is difficult and expensive to employ. On the other hand, nanosecond lasers have been used extensively to study shocked ${ }^{3}$ and warmdense matter. ${ }^{4}$ Many of these laser facilities are equipped with high intensity, short pulse lasers that can be used concurrently with the nanosecond laser pulse.

The use of high power, high intensity laser beams to accelerate protons has been well studied. ${ }^{5-9}$ In 2004 Lancaster et al., ${ }^{10}$ according to our knowledge, were the first to show the conversion of these laser-generated protons into neutrons by directing these protons on a $\mathrm{LiF}$ slab and taking advantage of the ${ }^{7} \mathrm{Li}(p, n)^{7} \mathrm{Be}$ reaction. More recently, Davis et al. ${ }^{11}$ showed numerical modeling of the ${ }^{7} \mathrm{Li}(d, x n)$ reaction using laser-accelerated deuterons. This work shows significant potential for creating a large amount neutrons with high energy $(>15 \mathrm{MeV})$ using a petawatt class laser.

In the NRS scheme, the laser-generated neutrons are moderated down to appropriate epithermal energies. The picosecond scale pulse length of the laser (as with the accelerator beam) and the ability to dope only a given section of the material, enables temporal and spatial effects (e.g., rarefaction) to be minimized. In this letter, it is shown that neutrons produced through $(p, n)$ reactions with laser accelerated protons are a route to performing NRS measurements in laser shocked materials.

The experiment was performed on the Titan Laser of the Jupiter Laser Facility at the Lawrence Livermore National Laboratory. The Titan laser is a Nd:glass laser with $\lambda_{\text {laser }}$ $=1054 \mathrm{~nm}$, used at best focus ( spot diameter of $7 \mu \mathrm{m}$ with $20 \%$ of energy) and shortest pulse length, 0.7 ps. The laser was focused on $\mathrm{Cu}$ foil of $25 \mu \mathrm{m}$ thickness, which accelerated high energy electrons in the forward direction setting up an electric field that accelerated a quasineutral proton beam from a hydrocarbon debris layer on the rear of the target. In phase I, these protons were incident on a radiochromic film (RCF) stack (see phase I of Fig. 1) to measure the proton distribution or, in phase II, they were incident on a $0.9 \mathrm{~mm}$ LiF converter foil (see phase II of Fig. 1). The LiF converter foil contains large cross-sections for $(p, n)$ reactions (see 


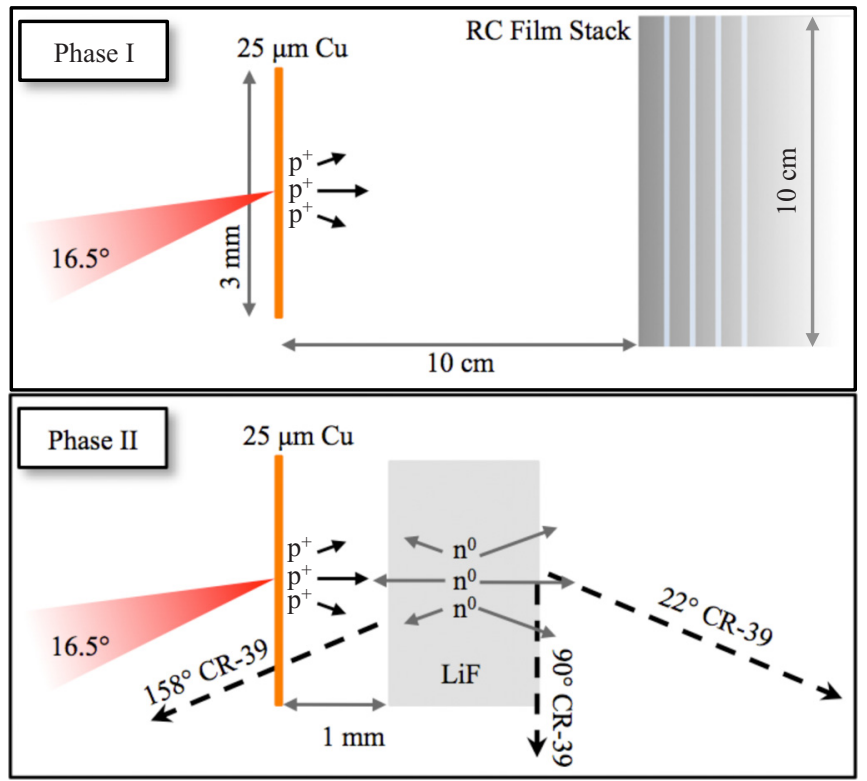

FIG. 1. (Color online) Experimental layout showing phase I, measurement of proton distribution using RCF and phase II showing neutron production through ${ }^{7} \mathrm{Li}(p, n)^{7} \mathrm{Be}$ reactions and neutron measurement using CR-39.

Fig. 4 below) which generated neutrons that were detected on absolutely calibrated CR-39 detectors at three different angles.

In phase I, RCF stacks were used to characterize the proton beam distribution. RCF is an absolutely calibrated film ${ }^{12}$ which contains an organic dye that increases in opacity with exposure to ionizing radiation. The RCF stack consists of alternating layers of $\mathrm{Al}$ filters and RCF. A total of ten sheets of RCF were used to give an energy resolution from 5.1 to $42.5 \mathrm{MeV}$. The lower limit was chosen to avoid signal contamination from other high energy ions accelerated off the foil. Note that the lower limit does not add significant error to our neutron predictions as the probability of neutron production at lower energies is quite small (see Fig. 5 below). The stopping of the protons and the energy that they deposited in each layer of film was calculated with the collisional Monte-Carlo code SRIM. ${ }^{13}$

In order to fit the data, a two temperature Maxwellian proton distribution was assumed, $d N / d \mathcal{E}$ $=\left(N_{1} / \sqrt{\pi \mathcal{E} T_{1}}\right) e^{-\mathcal{E} / T_{1}}+\left(N_{2} / \sqrt{\pi \mathcal{E} T_{2}}\right) e^{-\mathcal{E} / T_{2}}$, which is a function of proton energy $\mathcal{E}$. The parameters $N_{1}, N_{2}, T_{1}$, and $T_{2}$ representing proton number and proton temperature, respectively, were optimized to minimize the reduced $\chi^{2}$ fit to the data. This fit is compared with experimental data, shown in Fig. 2. The best fit is given by $T_{1}=2.9 \mathrm{MeV}, N_{1}=4.34$ $\times 10^{13}, T_{2}=10.27 \mathrm{MeV}$, and $N_{2}=5.44 \times 10^{12}$, with a reduced $\chi^{2}$ of 0.41 . The conversion efficiency into protons above $5.1 \mathrm{MeV}$ is $3.5 \%$ with is consistent with previous results. ${ }^{9}$ The largest error in the RCF comes from the batch to batch error which is $10 \% .^{12}$ The spectral distribution from above is shown in Fig. 3. Also shown is the proton spectrum inferred from a shot with similar laser parameters to illustrate shotto-shot variation.

In phase II, a LiF block of $0.9 \mathrm{~mm}$ was placed about $1 \mathrm{~mm}$ behind the rear of the $\mathrm{Cu}$ foil so that the proton beam

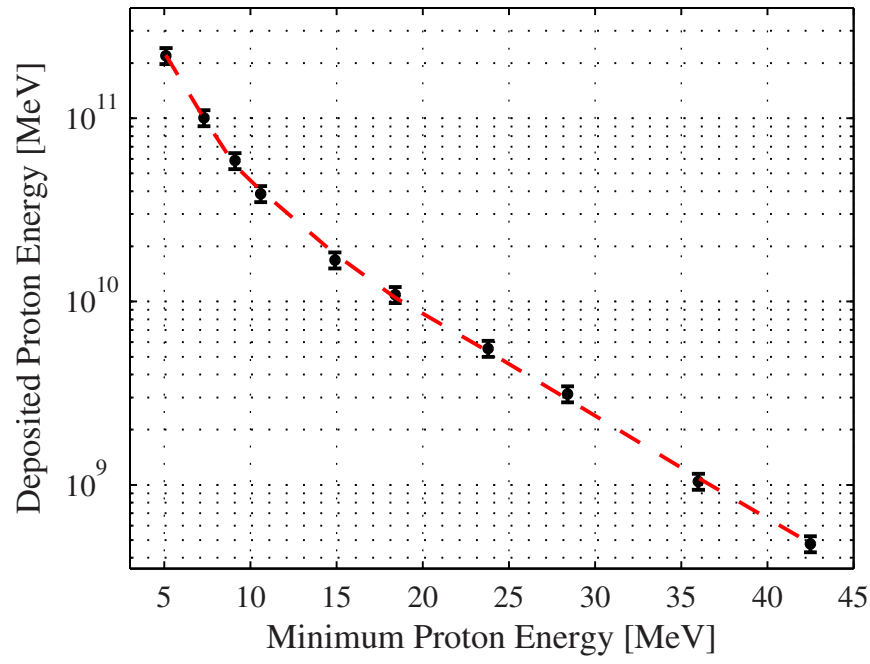

FIG. 2. (Color online) RCF data from a shot with $140 \mathrm{~J}$ on target and laser intensity of $1 \times 10^{20} \mathrm{~W} / \mathrm{cm}^{2}$. Each data point represents one layer of RCF, where the horizontal axis shows the minimum proton energy necessary to reach that layer. The experimental data are shown with circles $(\bullet)$. The dashed line is the best fit of a two temperature Maxwellian as described in the text.

was incident on the LiF converter. RCF was not used concurrently, as the proton beam was obstructed by the LiF converter. In order to record the neutron emission, three stacks of absolutely calibrated CR-39 detectors were placed at $22^{\circ}$, $90^{\circ}$, and $158^{\circ}$ from rear target normal at distances from 5 to $8.5 \mathrm{~cm}$ from the target. CR-39 is a plastic that can be damaged indirectly by neutrons through knock-on protons and has been used previously in laser generated proton experiments. ${ }^{10}$ Due to the sensitivity of CR-39 to protons, the CR-39 was shielded with $3.175 \mathrm{~mm}$ thick $\mathrm{Al}$ on the $90^{\circ}$ and $158^{\circ}$ pieces and $6.35 \mathrm{~mm}$ thick $\mathrm{Al}$ on the $22^{\circ}$ piece. The effectiveness of these filters was demonstrated by using RCF

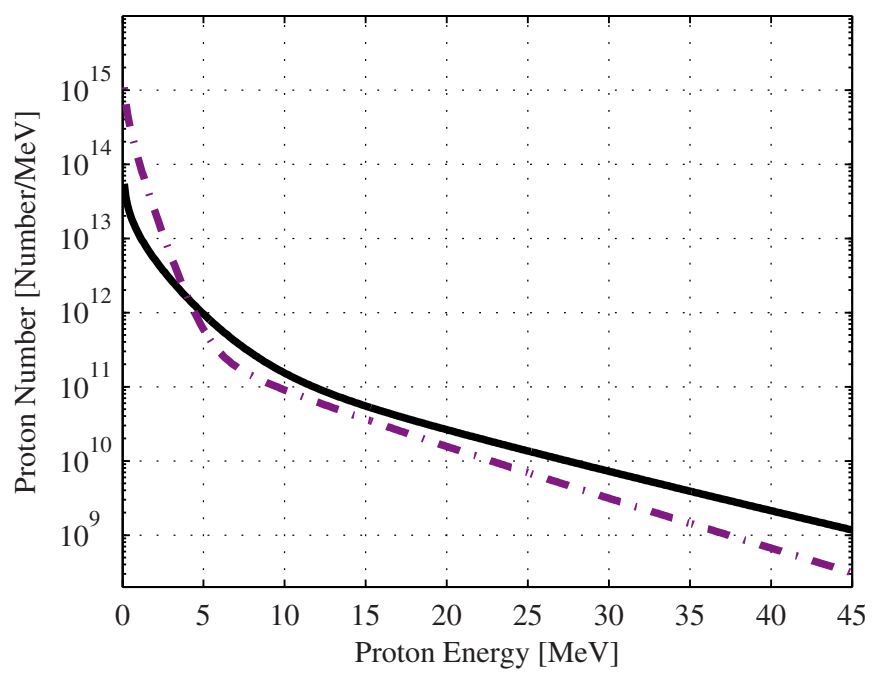

FIG. 3. (Color online) Proton spectra using a two-temperature Maxwellian distribution. The solid line represents the $140 \mathrm{~J}$ shot with data shown in Fig. 2 with proton number and temperature shown in the text. The dash-dotted line shows a shot of $120 \mathrm{~J}$ with otherwise the same laser parameters, illustrating the shot-to-shot variation in proton spectra. 


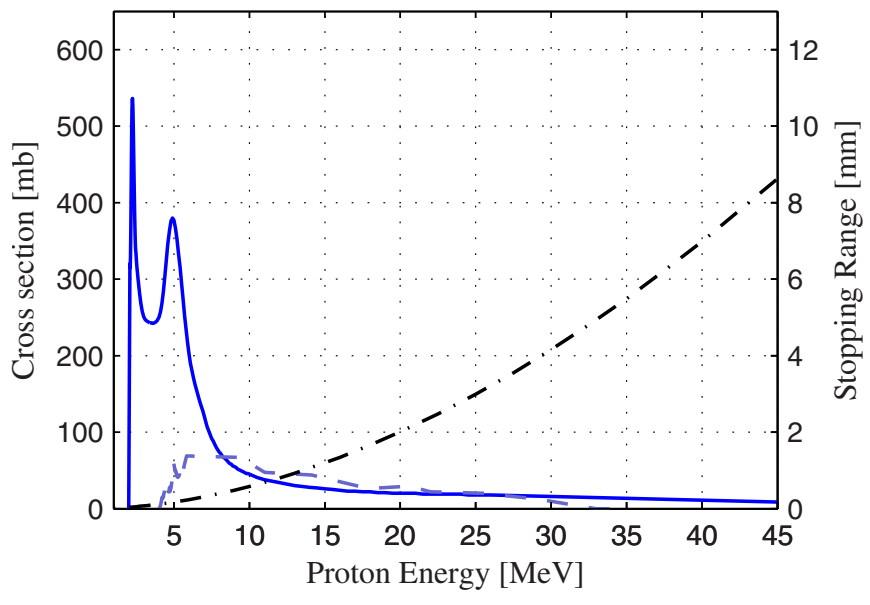

FIG. 4. (Color online) Plotted on the left axis, are cross-sections for ${ }^{7} \mathrm{Li}(p, n){ }^{7} \mathrm{Be}$ (solid line) and ${ }^{19} \mathrm{~F}(p, n){ }^{19} \mathrm{Ne}$ (dashed line) vs incident proton energy. The other stable isotope of $\mathrm{Li}$ is ${ }^{6} \mathrm{Li}$ which has no documented $(p, n)$ cross-sections and makes up only $7.5 \%$ of naturally occurring Li. On the right axis is the mean stopping range of protons in $\mathrm{LiF}$ as calculated with SRIM (dash-dotted line).

instead of CR-39 and verifying that there was no noticeable signal.

To determine the sensitivity of CR-39 to neutrons, a ${ }^{252} \mathrm{Cf}$ source was used. This is a reasonable assumption given that the CR-39 sensitivity is nearly constant with neutrons, $0.5-5 \mathrm{MeV}{ }^{14}$ The error in this measurement is calculated by adding in quadrature the variation in the background of unexposed CR-39 and the variation in the signal of the three CR-39 pieces within the detector stack.

The code DOWNSPEC (Ref. 15) was used to calculate the production of neutrons created in a given $(p, n)$ converter material by given energy protons. The code simultaneously calculated the stopping of incident protons as well as calculating their cross-sections for $(p, n)$ type reactions (see Fig. $4)$. This allows for determination of angular and energy resolved neutron yields. The code uses the continuous deceleration approximation model so it does not account for scattering or for energy broadening for a single energy incident proton.

A useful way to evaluate a material's ability to produce $(p, n)$ neutrons is to look at neutrons produced per incident proton at a given energy, as shown in Fig. 5. This is more useful than the cross-section alone because it also takes into account the stopping of protons in the material.

To predict the number of neutrons produced, the proton distributions (see Fig. 2) were used as inputs in DOWNSPEC, which gave angularly resolved neutron yields. These predicted yields are plotted in Fig. 6 against experimentally measured CR-39 yields. It is worth noting that there is shotto-shot variation even with the same laser energy. This fluctuation is common in short pulse laser experiments. ${ }^{8,9}$

In this letter, the capacity to predict and generate beams of $1.8 \times 10^{9}$ neutrons within a few picoseconds has been demonstrated. Using statistical error analysis, the predicted fractional uncertainty in NRS temperature measurements is $\Delta T / T=2 / \sqrt{N}$ where $N$ is the number of neutrons that are usable for NRS measurements (i.e., they pass through the

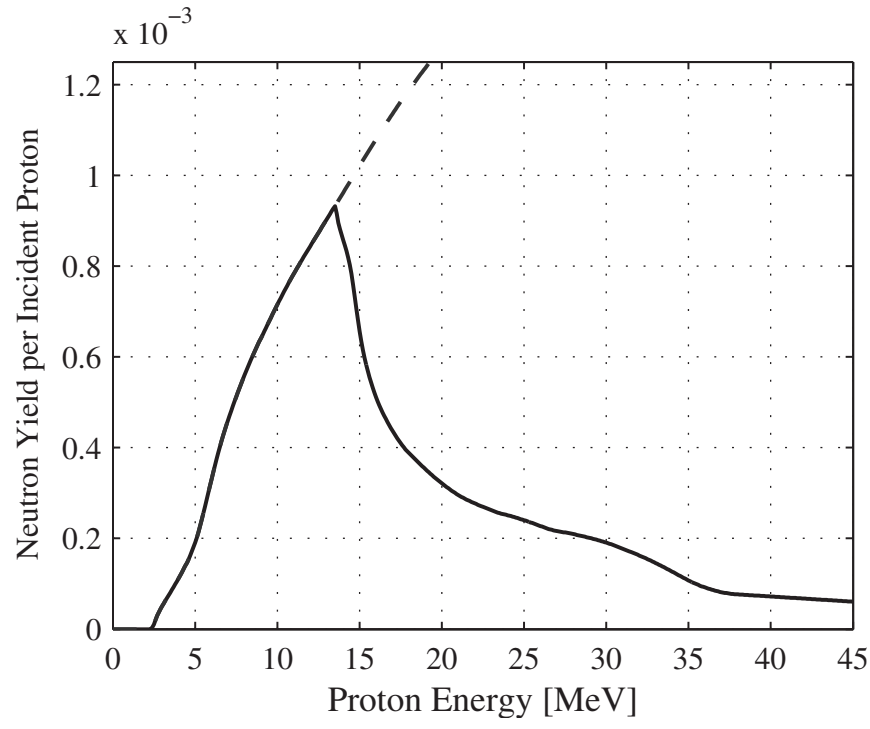

FIG. 5. Angularly integrated neutron production probability of protons of a given energy in LiF. The solid line is for $0.9 \mathrm{~mm}$ thickness, as used in the experiment, and the dotted line is for an infinite block of LiF.

target and are in the region of resonance), $\sqrt{N}$ is the statistical noise and 2 is the number of degrees of freedom (i.e., location and width of resonance). Thus for temperature measurement with $1 \%$ accuracy, $4 \times 10^{4}$ usable neutrons are required. The moderator setup must therefore capture the high energy neutrons, direct them toward the shocked target, and downshift their energy with an efficiency of $>2.2 \times 10^{-5}$.

The feasibility of NRS is highly dependent on the shock experiment to be measured. The choice of material being shocked or used as a dopant is significant due to the variation of incident neutron energies at which neutron resonances occur and the strength of these cross-sections. Other factors

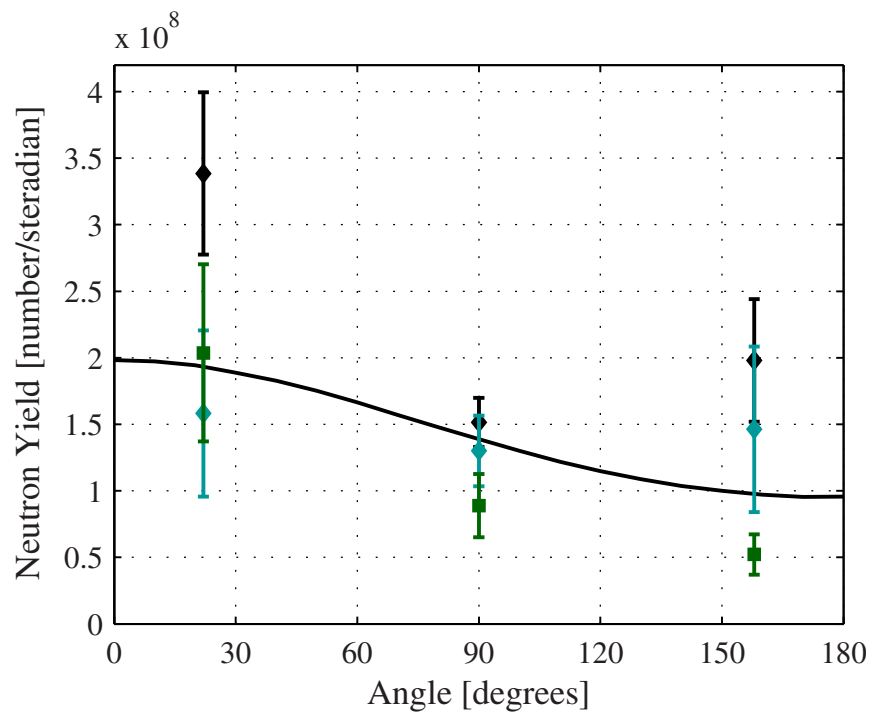

FIG. 6. (Color online) Experimental neutron yields (CR-39) plotted against predicted yields. Two shots of $127 \mathrm{~J}$ plotted with diamonds $(\checkmark)$ and one shot of $114 \mathrm{~J}$ is shown with squares $(\mathbf{D})$. The predicted yield, using a $140 \mathrm{~J}$ shot, is shown as the solid line. The error bars are due to variation of the background and signal of the CR-39 stacks. 
such as the dopant level and the solid angle subtended by the neutron detector during the shock experiment are also critically important. Preliminary analysis using a polyethylene moderator, in a geometry relevant to a laser facility, suggests that NRS can be successfully fielded as a temperature measurement technique in the laser environment.

To conclude, the viability of short-pulse laser produced epithermal neutrons as viable path for a NRS temperature diagnostic has been shown. Future experiments on the Titan laser will illustrate the capacity to moderate and collimate neutrons for NRS and to ultimately perform NRS temperature measurements on a sample ablatively loaded with a nanosecond laser pulse.

The authors acknowledge the staff of the Jupiter Laser Facility for their assistance in the execution of this work. This work performed under the auspices of the U.S. Department of Energy by Lawrence Livermore National Laboratory under Contract No. DE-AC52-07NA27344.

${ }^{1}$ V. W. Yuan, J. D. Bowman, D. J. Funk, G. L. Morgan, R. L. Rabie, C. E. Ragan, J. P. Quintana, and H. L. Stacy, Phys. Rev. Lett. 94, 125504 (2005).

${ }^{2}$ D. C. Swift, A. Seifter, D. Holtkamp, V. Yuan, D. Bowman, and D. A. Clark, Phys. Rev. B 77, 092102 (2008).

${ }^{3}$ R. F. Smith, J. H. Eggert, A. Jankowski, P. M. Celliers, M. J. Edwards, Y. M. Gupta, J. R. Asay, and G. W. Collins, Phys. Rev. Lett. 98, 065701 (2007).
${ }^{4}$ R. P. Drake, High-Energy-Density Physics: Fundamentals, Inertial Fusion, and Experimental Astrophysics (Springer, New York, 2006).

${ }^{5}$ A. P. Fews, P. A. Norreys, F. N. Beg, A. R. Bell, A. E. Dangor, C. N. Danson, P. Lee, and S. J. Rose, Phys. Rev. Lett. 73, 1801 (1994).

${ }^{6}$ E. Clark, K. Krushelnick, J. R. Davies, M. Zepf, M. Tatarakis, F. N. Beg, A. Machacek, P. A. Norreys, M. I. K. Santala, and I. Watts, Phys. Rev. Lett. 84, 670 (2000).

${ }^{7}$ R. A. Snavely, M. H. Key, S. P. Hatchett, T. E. Cowan, M. Roth, T. W. Phillips, M. A. Stoyer, E. A. Henry, T. C. Sangster, and M. S. Singh, Phys. Rev. Lett. 85, 2945 (2000).

${ }^{8}$ J. Fuchs, P. Antici, E. D'Humières, E. Lefebvre, M. Borghesi, E. Brambrink, C. A. Cecchetti, M. Kaluza, V. Malka, M. Manclossi, S. Meyroneinc, P. Mora, J. Schreiber, T. Toncian, H. Pépin, and P. Audebert, Nat. Phys. 2, 48 (2006).

${ }^{9}$ L. Robson, P. T. Simpson, R. J. Clarke, K. W. D. Ledingham, F. Lindau, O. Lundh, T. Mccanny, P. Mora, D. Neely, C. G. Wahlström, M. Zepf, and P. Mckenna, Nat. Phys. 3, 58 (2007).

${ }^{10}$ K. L. Lancaster, S. Karsch, H. Habara, F. N. Beg, E. L. Clark, R. Freeman, M. H. Key, J. A. King, R. Kodama, K. Krushelnick, K. W. D. Ledingham, P. McKenna, C. D. Murphy, P. A. Norreys, R. Stephens, C. Stoeckl, Y. Toyama, M. S. Wei, and M. Zepf, Phys. Plasmas 11, 3404 (2004).

${ }^{11}$ J. Davis, G. M. Petrov, T. Petrova, L. Willingale, A. Maksimchuk, and K. Krushelnick, Plasma Phys. Controlled Fusion 52, 045015 (2010).

${ }^{12}$ D. S. Hey, M. H. Key, A. J. Mackinnon, A. G. MacPhee, P. K. Patel, R. R. Freeman, L. D. V. Woerkom, and C. M. Castaneda, Rev. Sci. Instrum. 79, 053501 (2008)

${ }^{13}$ J. F. Ziegler, J. P. Biersack, and M. Ziegler, SRIM-The Stopping and Range of Ions in Matter (Lulu Press, Morrisville, NC, 2008).

${ }^{14}$ D. E. Hankins, S. G. Homann, and B. Buddemeier, Lawrence Livermore National Laboratory Report No. UCRL-53833, 1989.

${ }^{15}$ D. C. Swift, J. M. McNaney, D. P. Higginson, and F. N. Beg, Lawrence Livermore National Laboratory Report No. LLNL-TR-417474, 2009. 